\title{
Erratum to: Impact of 11C-choline PET/CT on clinical decision making in recurrent prostate cancer: results from a retrospective two-centre trial
}

Francesco Ceci • Ken Herrmann • Paolo Castellucci • Tiziano Graziani • Christina Bluemel • Riccardo Schiavina $\cdot$ Christian Vollmer • Sabine Droll • Eugenio Brunocilla •

Renzo Mazzarotto • Andreas K. Buck • Stefano Fanti

Published online: 19 September 2014

(C) Springer-Verlag Berlin Heidelberg 2014

Erratum to: Eur J Nucl Med Mol Imaging

DOI 10.1007/s00259-014-2872-x

Owing to errors in typesetting, the figures in this article as first published were in the wrong positions and therefore matched with the wrong legends. The article has now been corrected.

The online version of the original article can be found at http://dx.doi.org/ 10.1007/s00259-014-2872-x.

F. Ceci $\cdot$ P. Castellucci $\cdot$ T. Graziani $\cdot$ S. Fanti

Service of Nuclear Medicine, Policlinico S. Orsola-Malpighi,

University of Bologna, Bologna, Italy

F. Ceci • K. Herrmann · C. Bluemel · S. Droll • A. K. Buck

Department of Nuclear Medicine, Universitätsklinikum Würzburg,

Würzburg, Germany

R. Schiavina $\cdot$ E. Brunocilla

Department of Urology, Policlinico S. Orsola-Malpighi, University

of Bologna, Bologna, Italy

C. Vollmer

Department of Urology, Universitätsklinikum Würzburg, Würzburg, Germany

R. Mazzarotto

Service of Radiotherapy, Policlinico S. Orsola-Malpighi, University

of Bologna, Bologna, Italy

F. Ceci $(\bowtie)$

Policlinico S. Orsola-Malpighi, Azienda Ospedaliero-Universitaria

di Bologna, Via Massarenti, 9, 40138 Bologna, Italy

e-mail: francesco.ceci83@gmail.com 